\title{
An Interactive Dialogue Management System for Spoken Language
}

\author{
Manal Ali Alqahtani \\ Master in Information system, Shaqra University, Saudi Arabia \\ Email: manal.it1@gmail.com
}

\begin{abstract}
:
The spoken dialogue system is one of the most important human-machine communication ways. Human-machine communication can be described as an interaction between the user and the computer. This field is full of research points, so it is considered a good attractive environment for many researchers. The spoken dialogue system is of great importance in the process of communicating commercial applications, and facilitating the connecting process between the human and machine which may take different faces. The main objective of this research will be building an interactive dialogue management system for spoken dialogue system in an ideal way, By answering the following main question: How can we build an interactive dialogue management system for spoken dialogue system in an ideal way has the ability to accomplish the Naturalness, Usability, Mixed initiative, Cooperativity, Robustness, and Exploration. This research will be a mixed-method research and will adopt a descriptive survey design in collecting information by Survey questionnaires, Interviews to a sample of the target population, and while secondary data will be found from books, journals, and The Internet. The most important conclusion of the research is the spoken dialogue system is to be less complexity and use uncertainty model; this way must be acceptable by the user and the system itself.
\end{abstract}

Keywords: Management system, Spoken Language, An Interactive Dialogue 


\section{Introduction:}

\subsection{Background of the Study:}

An interactive dialogue management system for spoken dialogue system controls the overall interaction between the system and the user and coordinates activities of all spoken dialogue system's components.

The spoken dialogue system is one of the most important human-machine communication ways. Human-machine communication can be described as an interaction between the user and the computer. This field is full of research points, so it is considered a good attractive environment for many researchers.

Human-machine communication has taken several ways, these ways different according to the nature of the system and even the user, for example, the communication through the input text, press buttons or choose from menus, all these ways are not suitable for several systems and require a user who is professional and well trained to have the ability to deal with these ways.

Dialogue system seeks to provide a natural conversational interaction between the user and the computer system in an intelligent behavior.

Human-machine dialogue, therefore, has great significance for both academic and commercial applications. Evidence for the latter can be found in the frequent use of Spoken Dialogue Systems (SDS) in applications such as access to information and services, interaction with robots, playing games and providing customer support (Blaise Thomson \& Steve Young,2015).

The dialogue management system is the brain of the spoken dialogue system, given the importance of the dialogue management system; the thesis will be concentrated around by addressing all the components of the spoken dialogue system.

The spoken dialogue system components are automatic speech recognition, natural language understanding, natural language generation and finally the speech synthesize. 


\subsection{Significance of the Study:}

The spoken dialogue system has significance academically and as a practical method of communicating commercial applications, so the dialogue management system for spoken language faces a lot of challenges. At the commercial level the challenges may be emerged from environment and the user requirements. So; it is important to build a system that has the ability to accomplish the following characteristics:

Naturalness: this means that freedom of communication i.e. to be natural - like human conversation.

Usability: The goal of usability is building machines that are usable by untrained users

Mixed initiative: each participant can take the initiative.

Co-operativity: system should try to satisfy the user's goals, even where they are not expressed directly.

While at the academic level, the challenges emerged from the developing process itself. The system must be able to be:

Robustness: The system should be able to process ill-formed input. At the acoustic level, this implies that variation in the acoustic characteristics of the speech signal due to extraneous conditions should have little or no degradation on the performance of the recognizer. At the language level, the users should be able to express themselves naturally and freely without trapped by constrains imposed by the language model. At the understanding level, the presence of disfluencies and recognition error should have no or little impact on the behavior of the system.

Maintaining robustness at these various levels is the key to the success of the spoken dialogue system in general. 
Exploration: The ability of the system to be extended to recognize the new user entry. Most of researches have been done for building several computational models to accomplish these requirements.

\subsection{Statement of Problem:}

The importance of spoken dialogue system emerged from the facilitating the connecting process between the human and machine which may take different faces. The easiest way is to spoke to the machine through the spoken dialogue system, the spoken dialogue system must to be natural, easy to use for both the professional and native users, mixed initiative, co-operative, robustness and finally exploration, The previous spoken dialogue systems faced a lot of problems; some of these systems concentrated on the speech recognition and language understanding, and didn't use uncertainty models for the management process, this may be lead to weak performance.

The other spoken dialogue systems may solve this problem, but cause high complexity.

This thesis will be tried to avoid these problems by proposed a new model for the management process, this model will be using Markov Decision Process (MDP), but the MDP supposes that the surrounded environment is ideal, so this research will help MDP to get the ideal environment.

\subsection{Research Questions:}

The main research question of this research study is:

How can we build an interactive dialogue management system for spoken dialogue system in an ideal way?

Following are the sub-questions derived from the main question:

1. How can we implement to Automatic speech recognition system, and what are the stages of implementation of this system? 
2. How is the application a proposed system which gathers the natural -language understanding and dialogue management?

3. How are established a natural language generation system, and how are the use of A Hidden Markov Model in this system?

4. How are built text to speech system, and how we can do a comparison between different signal smoothing techniques in order to get a sound in a natural way?

\subsection{Research Aims and Objectives:}

The main target of this research will be building an interactive dialogue management system for spoken dialogue system in an ideal way. The ideal way means that the spoken dialogue system is to be less complexity and use uncertainty model; this way must be acceptable by the user and the system itself. As a trial to get the optimal solution, this thesis will be presented a proposed model for the management process, this model uses a Markov Decision Process (MDP), the MDP supposes that the surrounded environment is ideal. So; this research operates in all phases of the spoken dialogue system, theses phases are the effect on the dialogue management. So the main objectives of this research will be accomplished in the following :

- Automatic speech recognition system will be implemented to recognize the speech of the user. This system will be received a sentence and segment it into words, then recognize these words, in order to improve the performance, Principal component analysis was used to reduce the feature vector.

- A proposed system which gathers the natural language understanding and dialogue management will be implemented.

- A natural language generation system will be established; this system used A Hidden Markov Model. 
Academic Journal of Research and Scientific Publishing | Vol 3 | Issue 27

Publication Date: 5-7-2021 ISSN: 2706-6495

- Text to speech system will be built, and to get better results a comparison study will do between different signal smoothing techniques in order to get a sound in a natural way.

\subsection{Terminology of study:}

\section{- Spoken dialogue system:}

The spoken dialogue system in general consists of many phases as illustrated in (fig1) these phases, which cooperate to build a real spoken dialogue system.

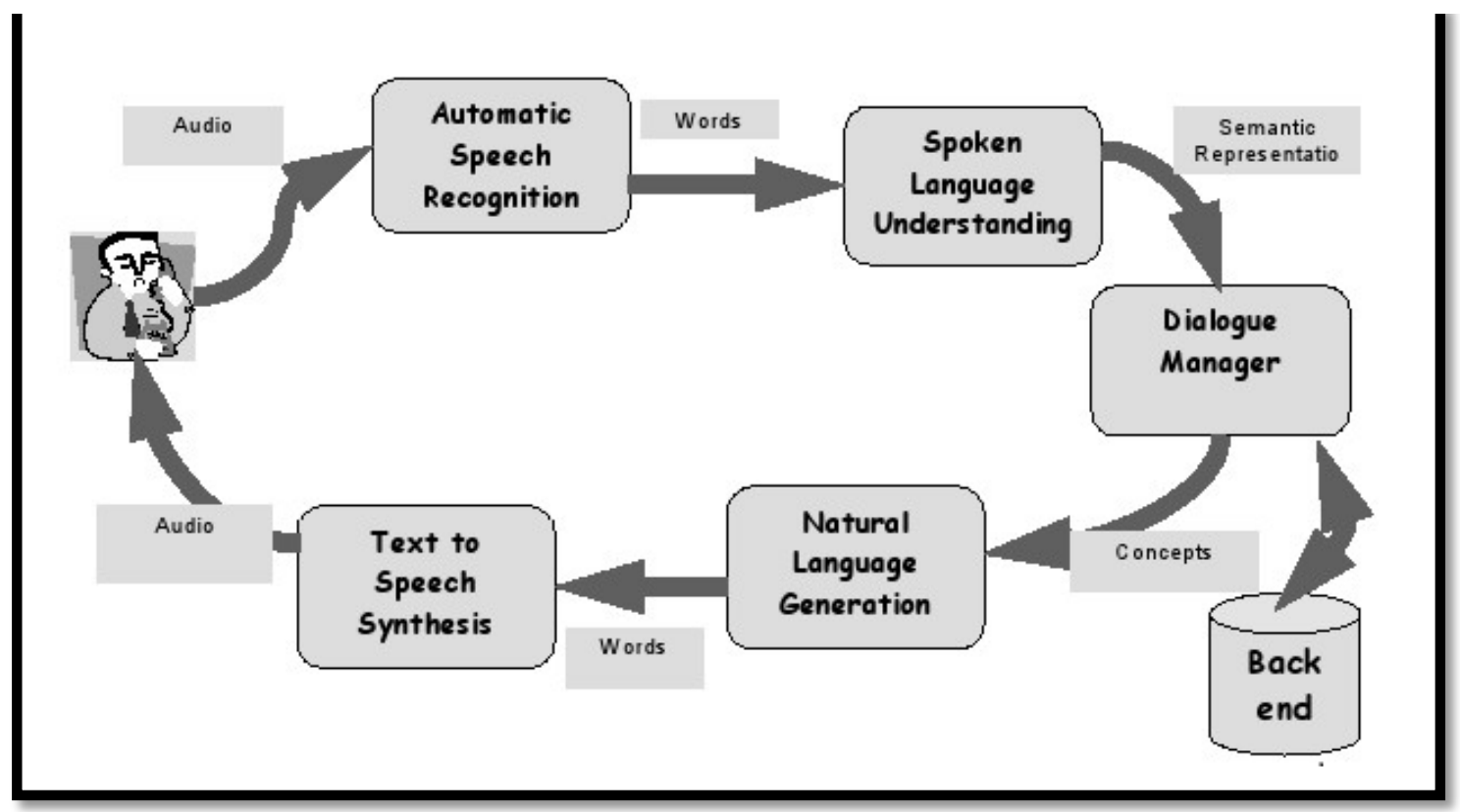

Fig (1) Spoken Dialogue System Components (Michael McTear, 2016).

\section{- Automatic Speech Recognition (ASR):}

Automatic speech recognition (ASR) is a very important phase as it transforms speech or the audio of emerged from the human to text. It has two basic approaches:

- Grammar-based ASR: The set of accepted phrases defined by regular/context-free grammars (i.e. language model in the form of a grammar), it is usually speaker independent (Michael McTear, 2016). 
- Dictation machine: Recognizes "any utterance" using N-gram language model, it is often speaker dependent (Michael McTear, 2016).

- Statistical approaches (Miroslav Melichar \& Martin Rajman, 2014).

\section{- Spoken Language Understanding (SLU):}

The SLU module or may be called natural language understanding (NLU) takes the output of ASR as input, and produce domain-specific semantic frames as output. These frames are intended to capture much of the meaning of the utterance, these semantic representations in forms like logical formula or Attribute (Kenji Sagae, Gwen Christian, David DeVault \& David R. Traum, 2013).

\section{- Dialogue Manger:}

The dialogue manager is the most important and effective phase, it coordinates all components activities; maintains the representation of the current state of the dialogue, communicates with external applications and decides about the next dialogue step.

The dialogue manager or dialogue management systems can be categorized in terms of the type of control offered and how the control is managed. Dialogue control may be system-led, user-led, mixed initiative or variable-initiative (Michael McTear, 2016).

\section{- Natural Language Generation (NLG):}

It is responsible for producing a textual utterance (so-called surface realization) from an internal (formal) representation of the answer. The surface realization can include formatting information such as speaking style, prosody, pauses, and background sounds (Miroslav Melichar \& Martin Rajman, 2014).

\section{Text to Speech Synthesis}

It is the final stage, which transforms the textual utterance received from the NLG to the audio form, and then the audio form will be transmitted to the user. 


\section{Literature Review}

The process of developing spoken dialogue system has taken several stages which growing gradually, in the past decade there are many activities in the area of spoken dialogue systems, largely due to government funding in the U.S. and Europe. By the late 1980's the DARPA spoken language systems program was initiated in the U.S., focusing on understanding speech input (James R. Glass ,1999). The DARPA considered the start point for a lot of systems as we will see. The research groups which took part each developed an understanding system for an air travel information service (ATIS) domain, undertook a joint data collection effort and underwent regular common evaluations (James R. Glass ,1999).

After that, a new DARPA program has begun which emphasizes dialoguebased interfaces incorporating both speech input and output technologies. One of the properties of this program is that participants are using common system architecture to encourage component sharing across sites. Participants in this program are developing both their own dialogue domains and a common complex travel task(Paul Ioan Fodor, 2005).In Europe, there have been several large research programs such as The Esprit SUNDIAL (Speech Understanding and Dialog) program sponsored research in four languages. This program promoted cooperation by requiring different sites to contribute components to a single multi-site system. In the more recent ARISE (Automatic Railway Information Systems for Europe) program. In addition to the research sponsored by these programs, there have been many other independent initiatives as well. For example, the Office Manager system developed at CMU provides voice access to application programs for the office of the future.

The Waxholm system provides ferry timetables and tourist information for the Stockholm archipelago (Joelle Pineau, Geoffrey Gordon \& Sebastian Thrun ,2006).This system concentrated on the speech recognition and language understanding and didn't use uncertainty models for the management process; this may be lead to weak performance. 
The direction of developing a telephone-based spoken dialog system comprises a telephone network interface to deliver calls into the system, a scheme for dialog authoring, a speech recognizer to accept requests from users, a method for comprehending requests, a means for response generation, a TTS engine for playing prompts and responses to the caller and a dialog manager to control the various components (Kinfe Tadesse Mengistu \& Andreas Wendemuth,2008).

In order to build a practical dialog system, it is essential to define the application domain of the system so that optimal recognition performance can be obtained by using proper acoustic, language and semantic models. The DARPA Communicator Systems, for instance, are constructed to play the role of a travel agent speaking by phone with a customer in a specific domain of Airline Travel Planning (Williams \& SJ Young ,2007), (Guy Shani, Ronen I. Brafman, \& Solomon E. Shimony)

Another instance of systems that a telephone-based is W99 which is a telephonebased spoken dialogue system for general workshop/conference services (Mazin Rahim, Roberto Pieraccini, Wieland Eckert, Esther Levin, Giuseppe Di Fabbrizio, Giuseppe Riccardi, Chih-mei Elaine Lin, \& Candy Kamm, 2009).

The University of Colorado (CU) Communicator is an interactive dialogue system for an airline, hotel, and rental car information (Bryan Pellom, Wayne Ward\& Sameer Pradhan ,2009). The CU Communicator integrates speech recognition, synthesis and natural language understanding technologies using the DARPA Hub Architecture to allow users to converse with an automated travel agent. During a typical telephone-based interaction, users can retrieve up-to-date travel information such as flight schedules, pricing, along with hotel and rental car availability. The system connects live over the web to get real up-to-date air travel, hotel, and rental car information.

Another spoken dialog system is The Let's Go Public (Antoine Raux, Brian Langner, Dan Bohus, Alan W Black,\& Maxine Eskenazi ,2010) it provides bus schedule information to the Pittsburgh population during off-peak times. 
The Let's Go bus information dialog system was a focus on making spoken dialog systems more accessible to the elderly and non-natives.Earlier efforts in this system were on speech synthesis modifications to aid the elderly understand synthetic speech and changes in speech recognition to aid non-natives. But experimental dialog systems are very different from commercial systems. So Many changes have been made to the Let's Go system to make it useful for the general public while retaining aspects that will allow continuing to experiment with dialog systems in general and for extreme user populations.

The new types of dialogue system appear such as The WHY2-ATLAS which is an intelligent tutoring system for qualitative physics that interacts with students via natural language dialogue (PamelaW. Jordan, Maxim Makatchev, Umarani Pappuswamy, Kurt VanLehn \& Patricia Albacete , 2011) it is for supporting a wider range of pedagogical strategies and for achieving a deeper understanding.

Most of the previous systems depend on the finite state and handcrafted approaches; these approaches don't support the user's incremental exploration and also may cause a fragile system especially in noisy environment. So another generation of dialogue system appears such as Voice-Rate is an experimental dialog system through which a user can call to get product information (Zhifei Li, Patrick Nguyen\& Geoffrey Zweig ,2013).

This algorithm uses a POMDP framework, which is probabilistic and captures uncertainty in speech recognition and user knowledge.

Simulation results show that the POMDP system performs significantly better than a deterministic baseline system in terms of both dialog failure rate and dialog interaction time. As a result of the increased use of the web-based application, new tools, and languages used in the implementation of spoken dialogue system such as the ate-A-Course system which implemented in VoiceXML (Amanda Stent, Svetlana Stenchikova \& Matthew Marge,2015). 
Also in (Lawrence Cavedon, 2016) the core of the system is the VoiceXML platform OptimTalk, OptimTalk is preferred to other voiceXML interpreters such as OpenVXI because it consists of not only a VoiceXML Interpreter but also a CCXML interpreter, and other abstract interfaces for the integration of ASR engine, TTS engine, and the telephony interface.

The important of Current spoken dialogue system emerged from the facilitating the connecting process between the human and machine; which may take different faces. The easiest way is to spoke to the machine through the spoken dialogue system, which must be natural, easy to use for both the professional and native users, mixed initiative, co-operative, robustness and finally exploration which means that the system able to be extended to recognize new user entry. But, the spoken dialogue system as a product may face a lot of problems, which may be partially solved in previous products. These solutions concentrate on the dialogue management as it considered a critical phase in the whole development process.

\section{Research Methodology:}

This chapter describes the research and the methodology that will be applied to carry out the study. It discusses the research design, data collection, data analysis, study period and mechanisms to assure the quality of the study. It also describes the nature and source of data sample size. The methods adopted for data collection are also illustrated, together with reliability and validity of the research instruments methods of data analysis and ethical consideration.

\subsection{Study design:}

This research will be a mixed-method research and will adopt a descriptive survey design in collecting information by administering questionnaires to a sample of the target population. The study will be aimed at collecting information from the respondents, regarding the ability of the proposed Dialogue system to provide a natural conversational interaction between the user and the computer system in an intelligent behavior. 
The investigator will use both the primary and the secondary data. Primary data will be obtained using questionnaires while secondary data will be found from books, journals, and The Internet.

\subsection{Study population and Sampling:}

The target population will form the basis of the respondents who will contribute to answering the survey questions. The study targets individuals who work in institutions such as service providers who have implemented the technologies being studied. The areas of the study were derived from the whole population, either developing the system, participating in its installation in an information, and communications environment or using these technologies.

\subsection{Data Collection Methods:}

The main data collection instruments to be used include the following:

1. Survey questionnaires.

\section{Interviews}

Survey Questionnaires have an advantage of achieving rapid contact with many people. It will be very useful for this research project to obtain responses to the diverse indicators requiring consultations with specific populations. (Engineers communications, electronics engineers, and information technology specialist, and other experts working in service providers)

The interview procedure is a shedding light on research process through an informal conversation. As part of this research project, an interview guide will be drawn to make the interviews semi-structured. Interview questions will be written down, and the interviewers will be trained so that they truly understand the subject matter as well the responses they receive.

\subsection{Instruments:}

To reach the optimal solution, some practical solutions will be applied in this study: 
1- Building Automatic speech recognition (ASR), the automatic speech recognition is responsible for receiving the sentences from the user; segmenting the sentence into words; building a model for each word, and finally using the PCA for the reduction of the vector's feature in order to get better results.

2- Developing new dialogue management (DM) model which co-operating with natural language understanding to manage the all phases in the spoken dialogue system

3- Implementing natural language generation (NLG) based and hidden markov model (HMM).

4- Applying spectral smoothing techniques on the text to speech which using the concatenation approach in order to get a natural voice.

\subsection{Data Analysis Methods:}

Quantitative analyses will be appropriate for this project with a bit of use qualitative methods as well. Codes will be assigned to qualitative variables such as reliability, and qualitative data analysis will be carried out using coding strategy. Content study will be used to analyze the substantial body of data collected in this study with the following procedure:

1. Reading of collected data

2. Definition of classification categories for the collected data.

3. The categorization of the collected data.

4. Quantification and statistical treatment of data.

5. The scientific description of the studied cases.

6. The interpretation of results.

For quantitative data analysis, both the descriptive and the inferential statistics will be done to better understand the ability of the proposed Dialogue system to provide a natural conversational interaction between the user and the computer system in an intelligent behavior. 


\subsection{Mechanisms to Assure Quality of the Study:}

\section{- Control of Bias:}

The researcher will make sure that the research study is objective, independent and balanced. The researcher believes that objectivity is critical to the success of scientific analysis. Thus, the researcher will actively work to keep a neutral and objective research environment, don't ask leading questions and tabulate results with respect to the alternative hypothesis.

\section{- Use accurate and most reliable data:}

To assure that the research study is useful, informative and understandable data and information of the best quality will be used. The data and information used in the research study will be validated from multiple sources and it will be assured that it is reliable and accurate.

\section{Summary:}

An interactive dialogue management system for spoken dialogue system controls the overall interaction between the system and the user and coordinates activities of all spoken dialogue system's components. The spoken dialogue system is one of the most important human-machine communication ways.

Dialogue system will be sought to provide a natural conversational interaction between the user and the computer system in an intelligent behavior.

The spoken dialogue system will consist of many phases; the most important one is the dialogue management, which is the core of the system, so the main target of this research is to build an interactive dialogue management system for spoken dialogue system in an ideal way.

The ideal way means that the spoken dialogue system is to be less complexity and use uncertainty model; this way must be acceptable by the 
user and the system itself. As a trial to get the optimal solution, this thesis presents a proposed model for the management process, this model uses a Markov Decision Process (MDP), the MDP supposes that the surrounded environment is ideal. So; this research operates in all phases of the spoken dialogue system, theses phases are an effect on the dialogue management.

So the main tasks of this research will be accomplished in the following steps:

- Automatic speech recognition system will be implemented to recognize the speech of the user. this system receives a sentence and segment it into words, then recognize these words, in order to improve the performance, the Principal component analysis will be used to reduce the feature vector.

- A proposed system which gathers the natural language understanding and dialogue management will be implemented.

- A natural language generation system will be established; this system used A Hidden Markov Model.

- Text to speech system will be built, and to get better results a comparison study will be done between different signal smoothing techniques in order to get a sound in a natural way.

\section{References}

Amanda Stent, Svetlana Stenchikova and Matthew Marge, "DIALOG SYSTEMS FOR SURVEYS: THE RATE-A-COURSE SYSTEM ", Computer Science Department, Stony Brook University, Stony Brook, NY 11794-4400 , 2015.

Anna Hjalmarsson ," Utterance Generation in Spoken Dialogue Systems", www.ida.liu.se/labs/nlplab/gslt/dsvt06/AnnaHj5.pdf, last access October 2013.

Antoine Raux, Brian Langner, Dan Bohus, Alan W Black,and Maxine Eskenazi ," Let"s Go Public! Taking a Spoken Dialog System to the RealWorld", 
INTERSPEECH 2005, pages 885-888 , September , 4-8, Lisbon , Portugal,2010.

Bachu R.G., Kopparthi S., Adapa B. and Barkana B.D., "Separation of Voiced and Unvoiced using Zero crossing rate and Energy of the Speech Signal", Advanced techniques in computing sciences and software engineering, , pp 279-282, Springer science,2010.

Blaise Thomson and Steve Young, "Bayesian update of dialogue state: A POMDP framework for spoken dialogue systems", University of Cambridge, Preprint submitted to Elsevier June 2015.

Bryan Pellom, Wayne Ward,and Sameer Pradhan, "The CU Communicator:An Architecture for Dialogue Systems" , Center for Spoken Language Research ,University of Colorado, Boulder, Colorado 80309-0594, USA, 2009.

Carlos Henrique Costa Ribeiro, "A Tutorial on Reinforcement Learning Techniques

http://www.ppgia.pucpr.br/ fabricio/ftp/Aulas/Mestrado/AS/ArtigosApresentacoes/Aprendizagem\%20por\%20Reforco/TAIC-tutorial_RL.pdf, march 2013.

Deepika Singh and Parminder Singh," Removal of Spectral Discontinuity in Concatenated Speech Waveform", International Journal of Computer Applications (0975 - 8887) Volume 53- No.16, September 2012.

Guy Shani, Ronen I. Brafman, and Solomon E. Shimony," Prioritizing Point-Based POMDP Solvers",Department of Computer Science, Ben-Gurion University, Beer-Sheva, Israel ,2007.

Ingmund Bjørkan," Speech Generation and Modification in Concatenative Speech Synthesis", PHD thesis, Norwegian University of Science and Technology,2010. 
James R. Glass ," CHALLENGES FOR SPOKEN DIALOGUE SYSTEMS ", Spoken Language Systems Group, MIT Laboratory for Computer Science, Cambridge, MA 02139,1999.

Joelle Pineau , Geoffrey Gordon and Sebastian Thrun ," Anytime Point-Based Approximations for Large POMDPs" , Journal of Artificial Intelligence Research 27 (2006) 335-380 , AI Access Foundation and Morgan Kaufmann Publishers, 2006.

Kenji Sagae, Gwen Christian, David DeVault and David R. Traum, "Towards Natural Language Understanding of Partial Speech Recognition Results in Dialogue Systems", Proceedings of NAACL HLT 2009: Short Papers, pages 53-56, June 2013.

Kinfe Tadesse Mengistu and Andreas Wendemuth,"Telephone-Based Spoken Dialog System Using HTK-based Speech Recognizer and VoiceXML ", Cognitive Systems Group, Otto-von-Guericke Universit“at, 39106 Magdeburg, Germany, 2008.

Lawrence Cavedon, "Dialogue, Dialogue Modeling, and Dialogue Systems", National ICT Australia Victoria Research Lab, 2016.

Mahwash Ahmed and Shibli Nisar," Text-to-Speech Synthesis using Phoneme Concatenation", International Journal of Scientific Engineering and Technology, Volume No.3 Issue No.2, pp : 193 - 197,2014.

Mazin Rahim, Roberto Pieraccini, Wieland Eckert, Esther Levin, Giuseppe Di Fabbrizio, Giuseppe Riccardi, Chih-mei Elaine Lin, and Candy Kamm, "W99 - A SPOKEN DIALOGUE SYSTEM FOR THE ASRU'99 WORKSHOP", AT\&T Labs- Research , 2009.

Michael McTear," Spoken Language Understanding for Conversational Dialog Systems", IEEE/ACL 2016 Workshop on Spoken Language Technology\Aruba, December 10-13, 2016. 
Miroslav Melichar and Martin Rajman ,"Introduction to dialogue systems", ECOLE POLYTECHNIQUE FEDERALE DE LAUSANNE,2014.

Newton," Review of methods of Speech Synthesis", M-Tech Credit Seminar Report, Electronic Systems Group, EE Dept., IIT Bombay, November, 2011, http://www.ee.iitb.ac.in/student/ newton/research/seminar_report.pdf, access $4 / 2014$.

Oliver Lemon, " Learning what to say and how to say it: jointoptimisation of spoken dialogue management and Natural Language Generation", Computer Speech \& Language 25, 2 (2010) 210",DOI : 10.1016/j.csl.2010.04.005, 2010.

Olivier Pietquin,’Optimising Spoken Dialogue Strategies within the Reinforcement Learning Paradigm”, http://cdn.intechopen.com/pdfswm/682.pdf\#page=1\&zoom=auto,-164, 165 , December ,2013.

PamelaW. Jordan, Maxim Makatchev, Umarani Pappuswamy, Kurt VanLehn and Patricia Albacete, "A Natural Language Tutorial Dialogue System for Physics", American Association for Artificial Intelligence , 2011.

Paul Ioan Fodor, "Dialog Management for Decision Processes" , Stony Brook University, Stony Brook, NY 11720, USA,2005.

S. SARASWATHI and R. VISHALAKSHY," Design of Multilingual Speech Synthesis System”, Intelligent Information Management, 2, 58-64, 2010.

VerenaRieser, Simon Keizer, Xingkun Liu and Oliver Lemon(2011)," Adaptive Information Presentation for Spoken Dialogue Systems:Evaluation with human subjects", Proceedings of the 13th European Workshop on Natural Language Generation (ENLG), Association for Computational Linguistics,France, pages 102-109, September 2011.

Weihua Cao, Gang Chen, Xin Chen and Min Wu(2011), ”Optimal Tracking Agent: A New Framework for Multi-Agent ReinforcementLearning”, 2011 
Academic Journal of Research and Scientific Publishing | Vol 3 | Issue 27

Publication Date: 5-7-2021 ISSN: 2706-6495

International Joint Conference of IEEE TrustCom-11/IEEE ICESS-11/FCST11, DOI 10.1109/TrustCom.2011.182, 2011.

Weihua Cao, Gang Chen, Xin Chen and Min Wu, "Optimal Tracking Agent: A New Framework for Multi-Agent Reinforcement Learning”, 2011 International Joint Conference of IEEE TrustCom-11/IEEE ICESS-11/FCST-11 ，DOI 10.1109/TrustCom.2011.182, 2011.

Williams and SJ Young," Scaling POMDPs for Spoken Dialog Management", IEEE Audio, Speech and Language Processing, 15(7):2116-2129, 2007.

Zhifei Li, Patrick Nguyen, and Geoffrey Zweig ," Optimal Dialog in ConsumerRating Systems using a POMDP Framework" , Proceedings of the 9th SIGdial Workshop on Discourse and Dialogue, pages 104-111,Columbus, Association for Computational Linguistics. June 2013.

Copyright (C) 2021 Manal Ali Alqahtani, AJRSP. This is an open-access article distributed under the terms of the Creative Commons Attribution License (CC BY NC). Doi: doi.org/10.52132/Ajrsp.e.2021.274 